\title{
THE RELATION BETWEEN POST-SHOCK TEMPERATURE, COSMIC-RAY PRESSURE, AND COSMIC-RAY ESCAPE FOR NON-RELATIVISTIC SHOCKS
}

\author{
Jacco VinK $^{1}$, Ryo YAmazaki ${ }^{2}$, Eveline A. Helder ${ }^{1}$, And K. M. Schure ${ }^{1}$ \\ ${ }^{1}$ Astronomical Institute, Utrecht University, P.O. Box 80000, 3508 TA Utrecht, The Netherlands; j.vink@astro-uu.nl \\ ${ }^{2}$ Department of Physics and Mathematics, Aoyama Gakuin University, 5-10-1 Fuchinobe, Sagamihara, Kanagawa 252-5258, Japan \\ Received 2010 July 27; accepted 2010 August 25; published 2010 October 4
}

\begin{abstract}
Supernova remnants (SNRs) are thought to be the dominant source of Galactic cosmic rays. This requires that at least $5 \%$ of the available energy is transferred to cosmic rays, implying a high cosmic-ray pressure downstream of SNR shocks. Recently, it has been shown that the downstream temperature in some remnants is low compared to the measured shock velocities, implying that additional pressure supported by accelerated particles is present. Here we use a two-fluid thermodynamic approach to derive the relation between post-shock fractional cosmic-ray pressure and post-shock temperature, assuming no additional heating beyond adiabatic heating in the shock precursor and with all non-adiabatic heating occurring at the subshock. The derived relations show that a high fractional cosmic-ray pressure is only possible if a substantial fraction of the incoming energy flux escapes from the system. Recently, a shock velocity and a downstream proton temperature were measured for a shock in the SNR RCW 86. We apply the two-fluid solutions to these measurements and find that the downstream fractional cosmic-ray pressure is at least $50 \%$ with a cosmic-ray energy flux escape of at least $20 \%$. In general, in order to have 5\% of the supernova energy to go into accelerating cosmic rays, on average the post-shock cosmic-ray pressure needs to be $30 \%$ for an effective cosmic-ray adiabatic index of $\gamma_{\text {cr }}=4 / 3$.
\end{abstract}

Key words: acceleration of particles - cosmic rays - ISM: supernova remnants - shock waves

Online-only material: color figures

\section{INTRODUCTION}

Although there have been many theoretical and observational advances in understanding cosmic-ray acceleration by supernova remnant (SNR) shocks over the last decade, it is still not yet clear whether SNRs are capable of putting more than $5 \%$ of their energy into cosmic rays. This number is necessary in order to explain the cosmic-ray energy density in the Galaxy, given the size of the Galaxy, the supernova rate, and the cosmic-ray escape time (e.g., Ginzburg \& Syrovatskij 1967, for an early discussion).

The theoretical advances consist, among others, of a better understanding of magnetic field amplification by cosmic-ray streaming (e.g., Bell 2004) and major improvements in the selfconsistent modeling of efficient cosmic-ray acceleration (e.g., Blasi et al. 2005; Kang et al. 2009; Vladimirov et al. 2008). These simulations show that SNR shocks can transfer up to $\sim 80 \%$ of the kinetic energy flux entering the shock to cosmic rays.

On the observational side, progress has been made through $\mathrm{X}$-ray observations which show that many young SNRs are actively accelerating electrons up to $10-100 \mathrm{TeV}$, and they show that the magnetic fields must indeed be amplified (e.g., Vink \& Laming 2003; Völk et al. 2005; Bamba et al. 2005; Ballet 2006). Another major source of progress has been the coming of age of $\mathrm{TeV}$ gamma-ray astronomy. $\mathrm{TeV}$ gamma-ray observations have shown us that many young SNRs are TeV sources (e.g., Aharonian et al. 2001, 2004; Albert et al. 2007; Acciari et al. 2010). This proves that SNRs are capable of accelerating particles up to at least $100 \mathrm{TeV}$. Unfortunately, a correct interpretation of the emission mechanism, i.e., pion-decay or inverse Compton scattering, is necessary for understanding the fraction of energy contained by accelerated particles (see the review by Hinton \& Hofmann 2009).
Recent GeV gamma-ray observations with the Fermi-LAT show that, whatever the emission mechanism, even a bright young SNR like Cassiopeia A (Cas A) has only transferred $\sim 2 \%$ of its explosion energy to accelerated particles. The interpretation is not yet clear: are Cas A and other young SNRs beyond their peak in acceleration power, and did most of the highest energy particles escape? Or did they not yet reach their full potential as sources of cosmic rays? Or are young SNRs not the dominant sources of Galactic cosmic rays?

Recently, another method of measuring cosmic-ray acceleration efficiency has drawn attention. It consists of measuring the proton temperature behind SNR shocks that are suspected of being efficient cosmic-ray accelerators, in view of their high-velocity or multi-wavelength properties. Without cosmic-ray acceleration there exists a simple relationship between the post-shock plasma temperature and shock velocity. However, when a shock accelerates cosmic rays, less energy is available for plasma heating. The proton temperature can be deduced from thermal Doppler broadening of $\mathrm{H} \alpha$ line emission from behind fast shocks (Heng 2010). Note that, unlike the electron temperature, the proton temperature is for cosmic abundance close to or equal to the mean plasma temperature.

This method was used by Helder et al. (2009) for an X-ray synchrotron emitting shock in the SNR RCW 86. The X-ray synchrotron emission indicates that the shock is actively accelerating particles to $>10 \mathrm{TeV}$ energies. Moreover, RCW 86 is detected by HESS as a TeV gamma-ray source (Aharonian et al. 2008). Indeed, Helder et al. (2009) found that the plasma temperature is a factor of at least three lower than expected given the measured shock velocity. However, this value could not be directly translated into a fractional cosmic-ray pressure behind the shock, but was translated into a lower limit of a fractional cosmic-ray pressure of $\geqslant 50 \%$. This lower limit was derived 
using the Rankine-Hugoniot relations for a two-fluid shock (see also Vink 2008).

Here we explore the Rankine-Hugoniot relations for a twofluid shock further. We show that for a given fractional cosmicray pressure $w\left(\equiv P_{\mathrm{cr}} / P_{\text {tot }}\right)$, there is a unique cosmic-ray energy escape flux, $\epsilon_{\mathrm{esc}}\left(=F_{\mathrm{cr}} / \frac{1}{2} \rho_{0} V_{s}^{3}\right)$, associated with it that only depends on the overall Mach number of the shock.

In the next section, we present the derivation of the relation between $w, \epsilon$, and post-shock plasma temperature. In Section 3, we discuss this relation and its limitations in the context of twofluid models and cosmic-ray acceleration models, and we use the relations to derive the cosmic-ray pressure content for the northeastern region of RCW 86 and for the newly measured plasma temperature of the young Large Magellanic Cloud SNR 0509-67.5 (Helder et al. 2010).

\section{THE RELATION BETWEEN ESCAPE AND PRESSURE OF COSMIC RAYS}

Efficient particle acceleration by shock fronts leads to a shock structure that deviates significantly from a normal "one-fluid" shock (e.g., Drury \& Voelk 1981; Berezhko \& Ellison 1999; Blasi et al. 2005; Vladimirov et al. 2008; Drury et al. 2009; Kang et al. 2009; Reville et al. 2009): the particles diffusing ahead (=upstream) of the shock form a shock precursor that pre-compresses and slows down the gas flowing into the shock. The pre-compression caused by the precursor adiabatically heats the gas. The Mach number at the shock is, therefore, reduced with respect to the overall Mach number, as the shock velocity is reduced and the gas pressure upstream of the shock is increased with respect to a shock without a precursor. Additional heating of the gas may occur in the precursor due to non-adiabatic processes, such as Alfvénic heating (e.g., Vladimirov et al. 2008; Caprioli et al. 2008). In such a multi-fluid system, the shock that heats the plasma is called the subshock. In the limit of a one-fluid gas, the subshock is identical to the shock.

Here we follow a different approach then (Blasi et al. 2005; Vladimirov et al. 2008; Kang et al. 2009; Reville et al. 2009) in that we treat the whole system only thermodynamically, using a two-fluid approach, with the two components representing the thermal gas and a gas of accelerated particles (cosmic rays). For the moment, we neglect the possible influence of non-adiabatic heating in the shock precursor due to interactions between the gas and accelerated particles. Our approach is reminiscent of the work by Drury \& Voelk (1981) (see also Achterberg et al. 1984; Voelk et al. 1984; Drury et al. 2009) and is an extension of the work presented by Vink (2008) and Helder et al. (2009).

\subsection{The Dependence of Post-shock Cosmic-ray Pressure on the Overall and Subshock Compression Ratio}

Our starting point is the basic equations expressing conservation of mass, momentum, and energy flux. We evaluate these expressions for three distinct regions: (0) far upstream, where the presence of accelerated particles can be neglected, (1) in the shock precursor just upstream of the subshock, and (2) behind (downstream of) the shock. Mass flux conservation gives

$$
\rho_{0} v_{0}=\rho_{1} v_{1}=\rho_{2} v_{2}
$$

with $v$ being the velocity of the gas with respect to the shock. Note that $v_{0}=V_{s}$, the shock velocity in the observer's frame of reference. Momentum flux conservation can be expressed as

$$
P_{0}+\rho_{0} V_{s}^{2}=P_{1}+\rho_{1} v_{1}^{2}=P_{2}+\rho_{2} v_{2}^{2},
$$

with $P$ being the pressure.

At this point it is convenient to introduce the Mach number $M_{0}$ for the shock structure far upstream,

$$
M_{0}^{2} \equiv \frac{1}{\gamma_{g}} \frac{\rho_{0} V_{s}^{2}}{P_{0}},
$$

with $\gamma_{g}$ being the adiabatic index of the thermal particles. In addition, we introduce the compression ratios across the different regions 0,1 , and 2 :

$$
\chi_{1} \equiv \frac{\rho_{1}}{\rho_{0}}, \chi_{2} \equiv \frac{\rho_{2}}{\rho_{1}}, \chi_{12} \equiv \chi_{1} \chi_{2}=\frac{\rho_{2}}{\rho_{0}} .
$$

We now use the assumption that the thermal pressure in the precursor is only due to adiabatic heating in the precursor, i.e., $P_{1, \text { th }}=P_{0} \chi_{1}^{\gamma_{g}}$ (cf. Drury et al. 2009) and that across the subshock the pressure associated with the accelerated particles does not change (see Drury \& Voelk 1981; Achterberg 2004). The cosmic-ray terms therefore cancel each other in Equation (2), when considering the momentum flux from region 1 to 2 . In other words, the non-thermal particle pressure at the subshock is only relevant for reducing the Mach number, but leads otherwise again to the standard, one-fluid relation for shock compression with a reduced Mach number given by

$$
M_{1}^{2}=\frac{\rho_{1} v_{1}^{2}}{\gamma_{g} P_{1}}=\frac{\rho_{0} V_{s}^{2} / \chi_{1}}{\gamma_{g} P_{0} \chi_{1}^{\gamma_{g}}}=M_{0}^{2} \chi_{1}^{-\left(\gamma_{g}+1\right)} \text {. }
$$

Combining Equations (2)-(4), one finds for the total downstream pressure $P_{2}$ and the thermal pressure $P_{\mathrm{th}}$ :

$$
\begin{aligned}
& \frac{P_{2}}{\rho_{0} V_{s}^{2}}=\frac{P_{2, \mathrm{cr}}+P_{2, \mathrm{th}}}{\rho_{0} V_{s}^{2}}=\frac{1}{\gamma_{g} M_{0}^{2}}+\left(1-\frac{1}{\chi_{12}}\right), \\
& \frac{P_{2, \mathrm{th}}}{\rho_{1} v_{1}^{2}}=(1-w) \frac{P_{2}}{\rho_{1} v_{1}^{2}}=\frac{\chi_{1}^{\gamma_{g}+1}}{\gamma_{g} M_{0}^{2}}+\left(1-\frac{1}{\chi_{2}}\right),
\end{aligned}
$$

with the subscript cr referring to the cosmic rays. We have introduced here the symbol $w$ describing the fraction of the downstream pressure contributed by cosmic rays (cf. Vink 2008; Helder et al. 2009):

$$
w \equiv \frac{P_{2, \mathrm{cr}}}{P_{2, \mathrm{th}}+P_{2, \mathrm{cr}}}
$$

Using $\rho_{1} v_{1}^{2}=\rho_{0} V_{s}^{2} / \chi_{1}$ we can now derive an expression for the fractional cosmic-ray pressure for a given Mach number $M_{0}$ :

$$
w=\frac{\left(1-\chi_{1}^{\gamma_{g}}\right)+\gamma_{g} M_{0}^{2}\left(1-\frac{1}{\chi_{1}}\right)}{1+\gamma_{g} M_{0}^{2}\left(1-\frac{1}{\chi_{12}}\right)} .
$$

For high Mach number shocks, this equation simplifies to

$$
w=\frac{1-\frac{1}{\chi_{1}}}{1-\frac{1}{\chi_{12}}}=\frac{\chi_{12}-\chi_{2}}{\chi_{12}-1}
$$



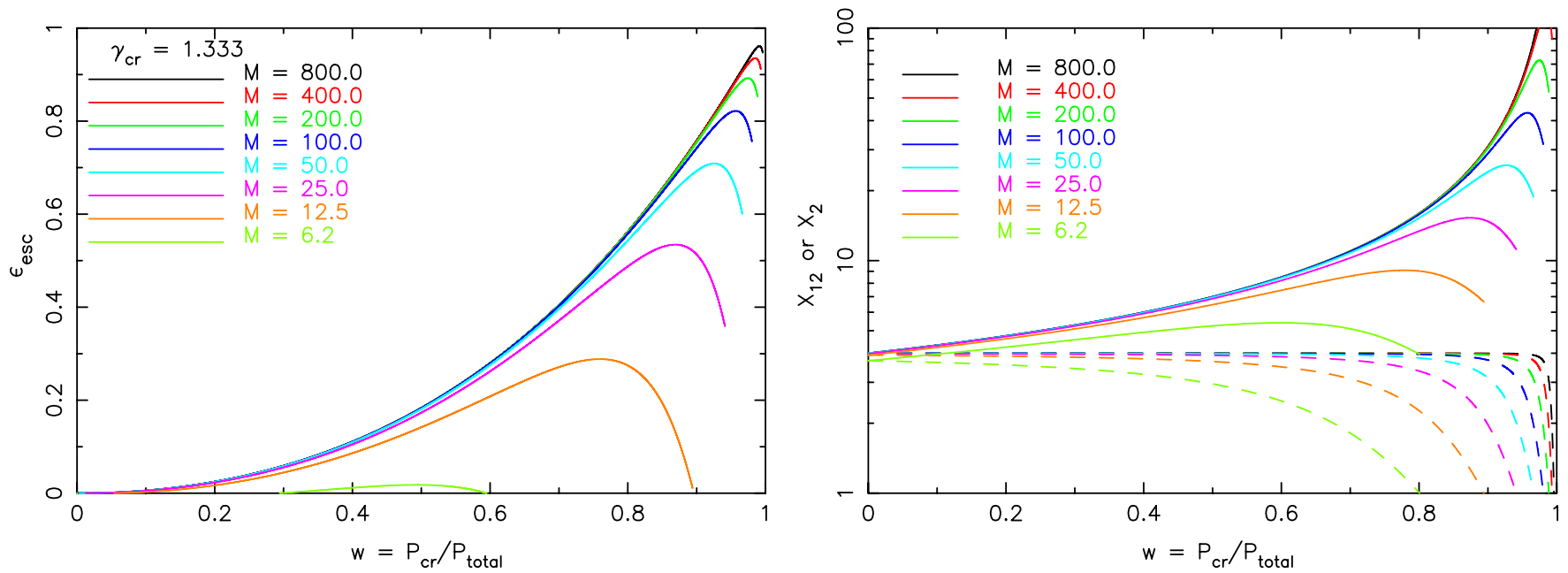

Figure 1. Left: relation between energy flux escape $\left(\epsilon_{\mathrm{esc}}\right.$, Equation (15)) and downstream fractional cosmic-ray pressure $(w$, Equation (9)) for an effective adiabatic index for the cosmic rays of $\gamma_{\mathrm{cr}}=4 / 3$ and for various values of the upstream Mach number $\left(M_{0}\right)$. Right: relation between overall compression ratio $\left(\chi_{12}\right.$, solid lines) and subshock compression ratio $\left(\chi_{2}\right.$, dashed lines).

(A color version of this figure is available in the online journal.)

The compression ratio of the subshock is given by the standard shock relation:

$$
\chi_{2}=\frac{\left(\gamma_{g}+1\right) M_{1}^{2}}{\left(\gamma_{g}-1\right) M_{1}^{2}+2},
$$

with $M_{1}$ being given by Equation (5).

Equations (5), (9), and (11) together show that there is a oneto-one relation between the compression ratio in the precursor and the downstream fractional cosmic-ray pressure for a given upstream Mach number $M_{0}$.

\subsection{The Escaping Energy Flux}

In order to determine the escaping energy flux carried away by particles diffusing away far upstream, we need to use the expression for conservation of energy flux across the shock, but with a modification in order to express the fact that energy flux may be lost from the system (e.g., Berezhko \& Ellison 1999):

$$
\left\{P_{2}+u_{2}+\frac{1}{2} \rho_{2} v_{2}^{2}\right\} v_{2}=\left\{P_{0}+u_{0}+\left(1-\epsilon_{\mathrm{esc}}\right) \frac{1}{2} \rho_{0} V_{s}^{2}\right\} V_{s},
$$

with $u=P /(\gamma-1)$ being the internal energy, and

$$
\epsilon_{\mathrm{esc}}=\frac{F_{\mathrm{cr}}}{\frac{1}{2} \rho_{0} V_{s}^{3}}
$$

being the escaping cosmic-ray energy flux, normalized to the total kinetic energy flux of the shock. Note that the escaping energy flux can only be taken out of the kinetic energy flux, as this is the only source of free energy. If we would have considered radiative losses, then the factor $\left(1-\epsilon_{\text {esc }}\right)$ should have been in front of $P_{0}$ as well, as the upstream thermal energy can also be radiated away.

Following Vink (2008) and Helder et al. (2009), we introduce for convenience

$$
G_{0} \equiv \frac{\gamma_{g}}{\gamma_{g}-1}, G_{2} \equiv w \frac{\gamma_{\mathrm{cr}}}{\gamma_{\mathrm{cr}}-1}+(1-w) \frac{\gamma_{g}}{\gamma_{g}-1} .
$$

Reordering the terms and using Equation (6) gives

$$
\epsilon_{\mathrm{esc}}=1+\frac{2 G_{0}}{\gamma_{g} M_{0}^{2}}-\frac{2 G_{2}}{\gamma_{g} M_{0}^{2} \chi_{12}}-\frac{2 G_{2}}{\chi_{12}}\left(1-\frac{1}{\chi_{12}}\right)-\frac{1}{\chi_{12}^{2}} .
$$

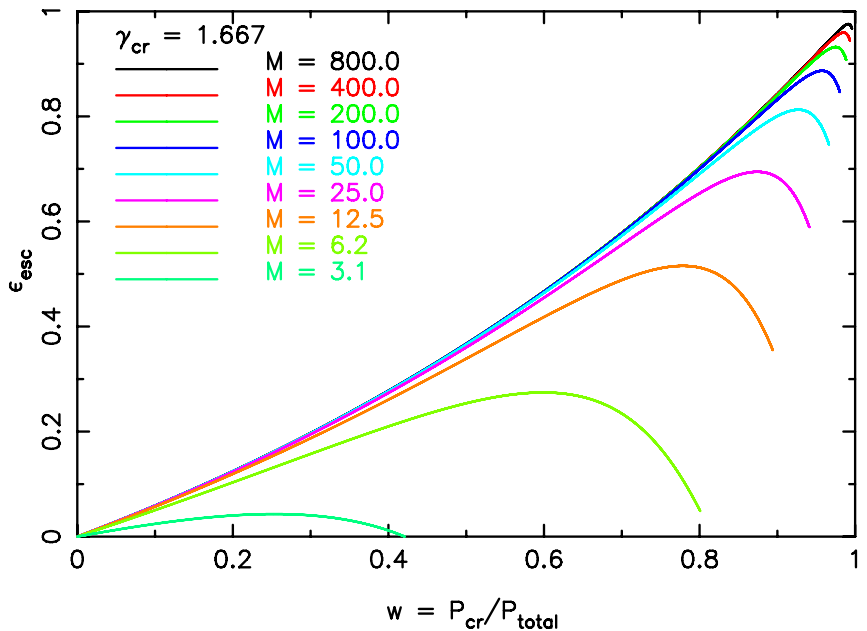

Figure 2. Left: same as the left panel of Figure 1 (left), but for an adiabatic index for the cosmic rays of $\gamma_{\mathrm{cr}}=5 / 3$

(A color version of this figure is available in the online journal.)

This equation completes the thermodynamic relation between the precursor compression ratio $\chi_{1}$ and downstream non-thermal pressure and overall energy flux escape. The resulting relation between $\epsilon_{\mathrm{esc}}$ and $w$ can be seen in Figure 1. For high Mach number shocks, the second and third terms can be omitted.

In the limit of $\gamma_{\text {cr }}=5 / 3$ and $M_{0}, M_{1} \rightarrow \infty$, one can show that the relation between $\epsilon_{\mathrm{esc}}$ and $w$ is well approximated by

$$
\frac{\epsilon_{\mathrm{esc}}}{w}=\left(1-\frac{1}{\chi_{12}}\right)^{2} \text {. }
$$

Since realistically $\gamma_{\mathrm{cr}}<5 / 3$ (see Section 3), Equation (16) serves as an upper bound on the escape flux.

For low Mach number shocks, there is a critical Mach number, $M_{0} \approx 6$, below which cosmic-ray acceleration cannot be thermodynamically supported for $\gamma_{\mathrm{cr}}=4 / 3$, as can be seen in Figure 1. For $\gamma_{\text {cr }}=5 / 3$ (Figure 2), cosmic-ray acceleration can be supported for lower Mach numbers, but there is still a limit, because $\epsilon_{\text {esc }}<0$ for $M_{0} \approx 2.5$. 

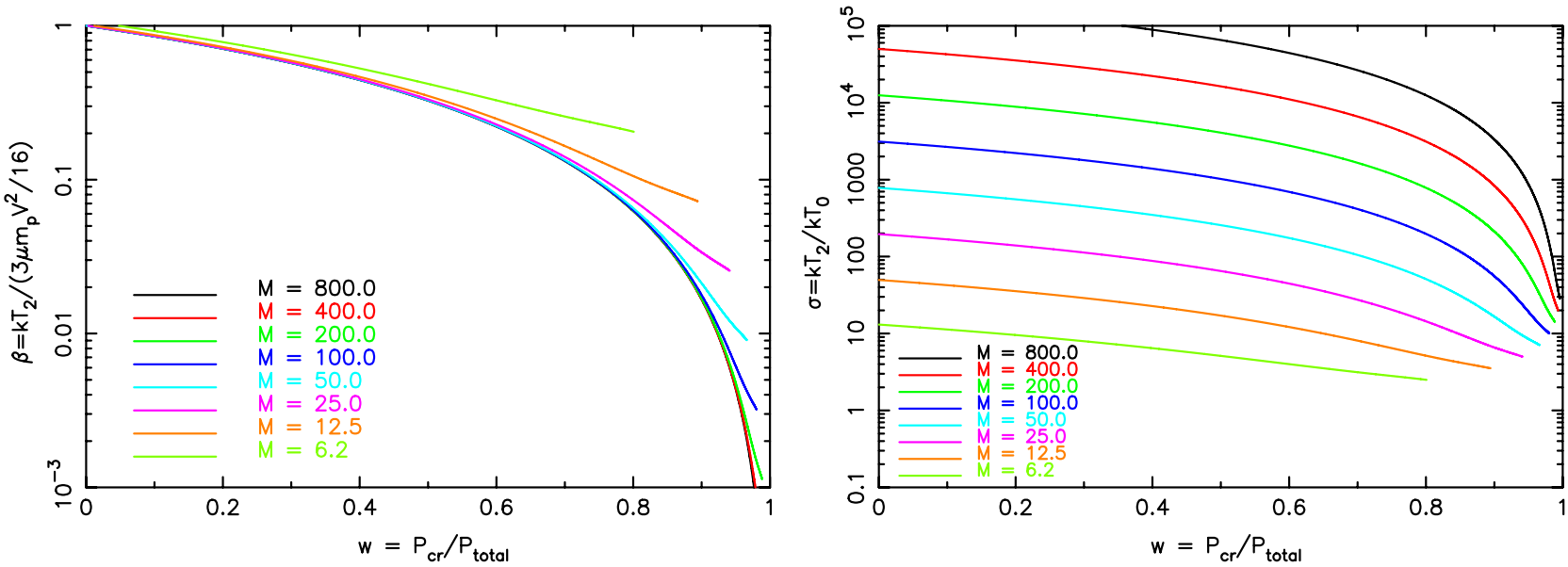

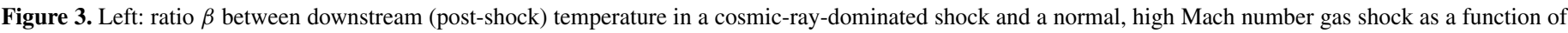
fraction downstream cosmic-ray pressure $w$. Right: ratio $\sigma$ between upstream and downstream temperatures as a function of $w$.

(A color version of this figure is available in the online journal.)

To summarize, taking $\chi_{1}$ as the principal variable and $M_{0}$ as an input parameter, one can calculate the subshock Mach number $M_{1}$ (Equation (5)) from which the subshock compression ratio follows (Equation (11)), and therewith the overall compression ratio $\chi_{12}=\chi_{1} \chi_{2}$. This can then be used to calculate $w$ (Equation (9)) and $\epsilon_{\text {esc }}$ (Equation (15)). We illustrate this in Figure 1.

\subsection{The Maximum Overall Compression Ratio}

Note that although for a given $w$ the escape flux $\epsilon_{\text {esc }}$ can be calculated, the opposite is not true. The reason is that $\epsilon_{\text {esc }}$ has a maximum. In fact, mathematically the curves beyond the maximum declines further than those indicated in Figure 1 (left), but this would correspond to an unphysical compression factor of $\chi_{2}<1$ at the subshock. The peak value in the overall compression ratio corresponds to a maximum in $\epsilon_{\text {esc }}$. The peak value can be found by differentiating the expression for the total compression ratio (see Equation (11)):

$$
\chi_{12}=\frac{\left(\gamma_{g}+1\right) M_{1}^{2} \chi_{1}}{\left(\gamma_{g}-1\right) M_{1}^{2}+2}=\frac{\left(\gamma_{g}+1\right) M_{0}^{2} \chi_{1}^{-\gamma_{g}}}{\left(\gamma_{g}-1\right) M_{0}^{2} \chi_{1}^{-\left(\gamma_{g}+1\right)}+2}
$$

with respect to $\chi_{1}$, and setting $d \chi_{12} / d \chi_{1}=0$. This gives

$$
\chi_{1}=\left(\frac{\gamma_{g}-1}{2 \gamma_{g}} M_{0}^{2}\right)^{1 /\left(\gamma_{g}+1\right)} .
$$

Inserting this in Equation (11) using Equation (5) shows that $\chi_{12}$ reaches a maximum for a subshock compression ratio of

$$
\chi_{2, \max }=\frac{\gamma_{g}}{\gamma_{g}-1}=5 / 2,
$$

with the numerical value valid for $\gamma_{g}=5 / 3$.

\subsection{The Downstream Temperature}

From Equations (6) and (9) or (7) it is possible to find an expression for the downstream temperature:

$$
\begin{aligned}
k_{\mathrm{B}} T_{2} & =P_{2, \mathrm{th}} / n_{2} \\
& =(1-w) \frac{1}{\chi_{12}}\left[\frac{1}{\gamma_{g} M_{0}^{2}}+\left(1-\frac{1}{\chi_{12}}\right)\right] \mu m_{\mathrm{p}} V_{s}^{2} \\
& =\frac{1}{\chi_{1} \chi_{12}}\left[\frac{\chi_{1}^{\gamma_{g}+1}}{\gamma_{g} M_{0}^{2}}+\left(1-\frac{1}{\chi_{2}}\right)\right] \mu m_{\mathrm{p}} V_{s}^{2},
\end{aligned}
$$

with $k_{\mathrm{B}}$ being the Boltzmann constant and $\mu$ being the mean mass per particle in units of the proton mass $m_{\mathrm{p}}$. This expression should be compared to the temperature expected behind a strong single-fluid shock:

$$
k_{\mathrm{B}} T_{2}=\frac{1}{\chi_{12}}\left(1-\frac{1}{\chi_{12}}\right) \mu m_{\mathrm{p}} V_{s}^{2}=\frac{3}{16} \mu m_{\mathrm{p}} V_{s}^{2},
$$

which can be obtained from Equation (20) by setting $\chi_{12}=4$ (valid for a strong shock with $\gamma_{g}=5 / 3$ ), $w=0$, and $M_{0} \rightarrow \infty$.

It is useful to define the ratio

$$
\beta=\frac{k_{\mathrm{B}} T_{2}}{\frac{3}{16} \mu m_{\mathrm{p}} V_{s}^{2}}
$$

between the downstream temperature in the presence of cosmicray acceleration and the expected temperature for a pure gas shock, as this is a quantity that can be related to existing measurements (cf., Helder et al. 2009, 2010). The behavior of $\beta$ is shown in Figure 3 (left). Note that Equation (20), like the expression for $w$ (Equation (9)), only relies on momentum conservation. As a result, there is a unique relation between $\beta$ and $w$. Since $w$ does not depend on the adiabatic index $\gamma_{\text {cr }}$ of the accelerated particles, $\beta$ does not depend on $\gamma_{\mathrm{cr}}$, and, therefore, $\beta$ does not depend on the energy distribution of the accelerated particles.

In addition, we define the ratio $\sigma=k_{\mathrm{B}} T_{2} / k_{\mathrm{B}} T_{0}$ between downstream and upstream temperatures, as it is a quantity used by Blasi et al. (2005) and Drury et al. (2009). The expression for this quantity is

$$
\begin{aligned}
\sigma=\frac{k_{\mathrm{B}} T_{2}}{k_{\mathrm{B}} T_{0}} & =(1-w) \frac{P_{2}}{\rho_{0} V_{s}^{2}} \frac{\gamma_{g} M_{0}^{2}}{\chi_{12}} \\
& =(1-w) \frac{1}{\chi_{12}}\left[1+\gamma_{g} M_{0}^{2}\left(1-\frac{1}{\chi_{12}}\right)\right] \\
& =\frac{1}{\chi_{1} \chi_{12}}\left[\chi_{1}^{\gamma_{g}+1}+\gamma_{g} M_{0}^{2}\left(1-\frac{1}{\chi_{2}}\right)\right] .
\end{aligned}
$$




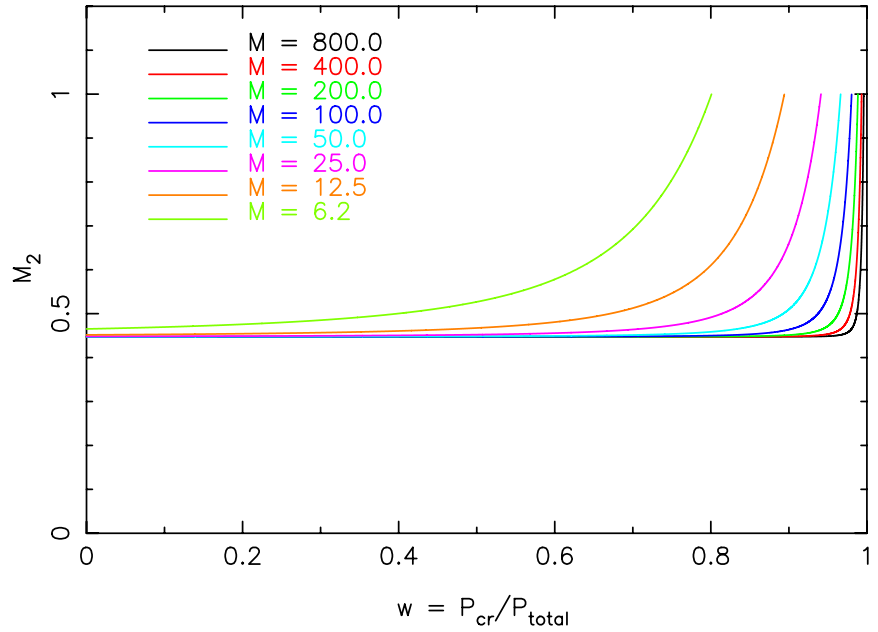

Figure 4. Downstream Mach number $M_{2}$ as a function of the fractional cosmicray pressure $w$.

(A color version of this figure is available in the online journal.)

The behavior of $\sigma$ as a function of $w$ is shown in the righthand panel of Figure 3. In addition, we show in Figure 4 the downstream Mach number $M_{2}$. This shows that for high $w$ the downstream Mach number rapidly approaches $M_{2}=1$. For $\chi_{2}=1$, i.e., a continuous shock, we have $M_{2}=1$, which leads to an unstable situation, as was already established by Drury \& Falle (1986).

\subsection{The Potential Influence of Magnetic Field Amplification}

As mentioned in Section 1, there is now observational evidence for magnetic field amplification by shocks in young SNRs. In our two-fluid approach, we ignore the effects of magnetic fields on the flow parameters, although we hope to come to this issue in the near future. For now, we offer an assessment of the magnitude of magnetic amplification on the cosmic-ray-dominated shocks.

We start by noting that the downstream magnetic field in several young SNRs is consistent with $\left(B_{2}^{2} / 8 \pi\right) / \rho_{0} V_{s}^{2} \approx 1 \%$ (Völk et al. 2005; Vink 2008). The question now is what is the perpendicular magnetic field pressure on the subshock compared to the ram pressure, i.e., what is $\left(B_{1, \perp}^{2} / 8 \pi\right) /\left(\rho_{1} v_{1}^{2}\right)$ ? This can be calculated using the fact that $B_{1, \perp}=B_{2, \perp} / \chi_{2}$ :

$$
\frac{B_{1, \perp}^{2} /(8 \pi)}{\rho_{1} v_{1}^{2}}=\frac{B_{2, \perp}^{2} /(8 \pi)}{\rho_{0} V_{s}^{2}}\left(\frac{\chi_{1}}{\chi_{2}^{2}}\right)=\frac{B_{2, \perp}^{2} /(8 \pi)}{\rho_{0} V_{s}^{2}}\left(\frac{\chi_{12}}{\chi_{2}^{3}}\right) .
$$

Since $B_{2, \perp}^{2}<B_{2}^{2}$ we can say that observations of young SNRs indicate that

$$
\frac{B_{1, \perp}^{2} /(8 \pi)}{\rho_{1} v_{1}^{2}} \lesssim 1 \%\left(\frac{\chi_{12}}{\chi_{2}^{3}}\right)
$$

From Figure 1 (right) we can see that for a large range of $w$ the factor $\chi_{12} / \chi_{2}^{3}$ is smaller than 1. Around the maximum of $\chi_{12}$ we have $\chi_{2}=2.5$. So in order to have $\left(B_{2}^{2} / 8 \pi\right) / \rho_{0} V_{s}^{2} \gtrsim 10 \%$, one needs $\chi_{12} \gtrsim 158$, which is larger than for any of the models depicted in Figure 1.

However, there has been a debate as to whether $B^{2} \propto \rho_{0} V_{s}^{2}$ (Völk et al. 2005) or $B^{2} \propto \rho_{0} V_{s}^{3}$ (Vink 2008; Tatischeff 2009). For the young SNRs, the precise proportionality does not change our conclusion very much, but in very young SNRs and/or for radio supernovae this may be relevant. In particular, for $\mathrm{SN}$
1993J, which was a bright radio supernova, magnetic fields as high as $50 \mathrm{G}$ have been inferred, and $B^{2} /(8 \pi) \sim 0.1 P_{\text {cr }}$ (e.g., Tatischeff 2009). At such a level, the magnetic fields may start to become dynamically important.

Apart from amplifying the magnetic field upstream of the shock, cosmic rays in the precursor may also give rise to nonadiabatic gas heating (e.g., Vladimirov et al. 2008; Caprioli et al. 2009). The effects of non-adiabatic heating will be similar to having a lower Mach number shock, i.e., non-adiabatic heating in the precursor limits the maximum overall compression ratio and limits the maximum possible fractional cosmic-ray pressure in the downstream region.

\subsection{The Entropy Change}

The entropy jump across a shock is given by the relation (e.g., Zeldovich \& Raizer 1966)

$$
\Delta S=\frac{3}{2} k_{\mathrm{B}} \ln \left(\frac{P_{2} \rho_{2}^{-\gamma_{g}}}{P_{0} \rho_{0}^{-\gamma_{g}}}\right)
$$

This neglects the entropy increase associated with the accelerated particles. Brown et al. (1995) calculated the entropy of non-thermal distributions, which, not surprisingly, always have entropy values below a thermal distribution. Moreover, they normalize the distribution to the number of particles involved. However, in reality, the cosmic-ray component may contribute a dominant fraction of the pressure, but the number of particles is always much smaller than the number of thermal particles. So neglecting the entropy of the cosmic rays is a good approximation.

Figure 5 (left) shows that when the fractional cosmic-ray pressure $w$ increases the jump in entropy decreases. The righthand panel is more interesting as it shows that for certain values of the escaping energy flux $\epsilon_{\mathrm{esc}}$ there are two possibilities for the jump in entropy. The maximum value of $\epsilon_{\mathrm{esc}}$ as a function of entropy change $\Delta S$ again occurs for the value for which $\chi_{12}$ has a maximum. One can speculate that whenever there are possible solutions to obtain a certain value for $\epsilon_{\mathrm{esc}}$ the nature chooses the one that offers the highest entropy jump. On the other hand, the escape has to be facilitated by the details of the acceleration process and particle spectrum, so one should not discount the low entropy branch too easily.

\section{DISCUSSION}

The solutions that we presented here for the two-fluid approach to shock with a cosmic-ray component give us a handle on estimating the effects of cosmic-ray acceleration on the postshock plasma temperature and, in addition, allow us to estimate the cosmic-ray escape. It may not be too surprising that there is a thermodynamic relation between the fractional cosmic-ray pressure $w$ and escape. The reason is that in order to have a shock jump one needs either viscous shock or one needs to lose energy from the system. The problem with a cosmic-ray component is that the accelerated particles do not cause a jump in entropy. Therefore, the increased overall shock compression has to be facilitated by energy escape.

This situation will change once additional heating in the precursor caused by cosmic-ray heating is introduced. This heating is presumably the result of dissipation of large-amplitude magnetohydrodynamic waves in the cosmic-ray precursor (Alfvénic heating); these waves are the result of cosmic-ray streaming 

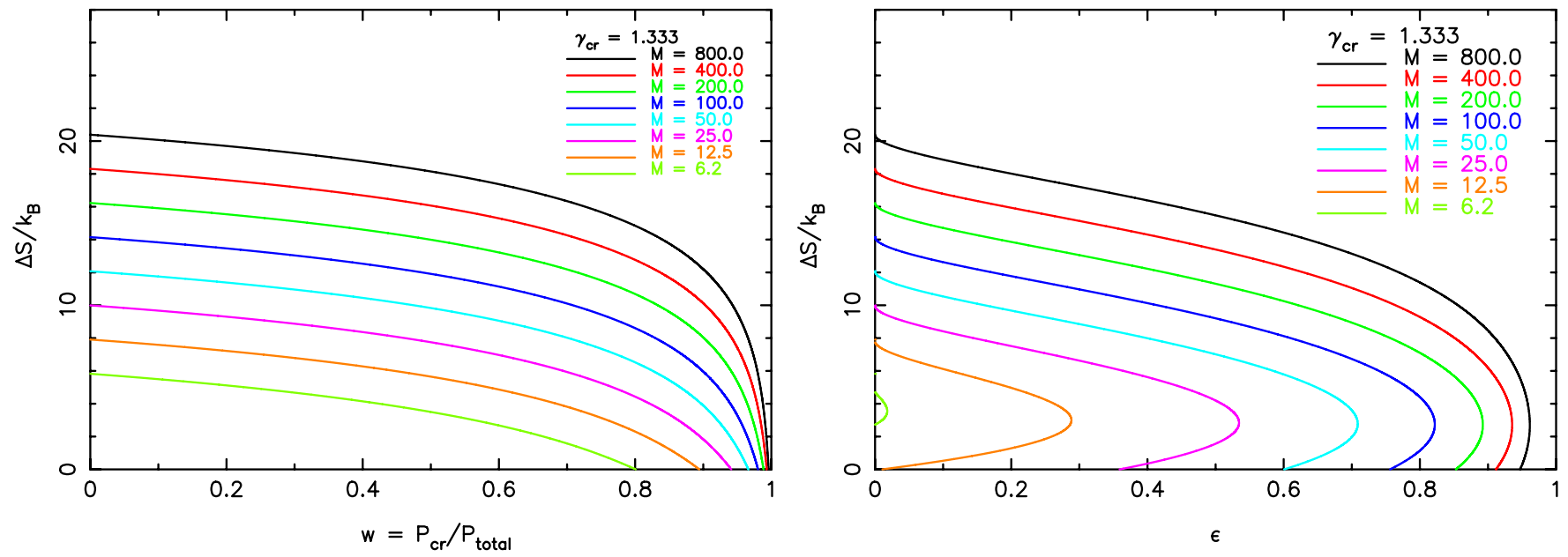

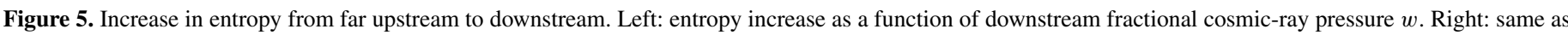
left, but as a function of escaping energy flux $\epsilon_{\text {esc }}$.

(A color version of this figure is available in the online journal.)

(Vladimirov et al. 2008; Caprioli et al. 2009). The most important effect of heating in the precursor will be that the Mach number at the subshock $M_{1}$ will be decreased. So we expect that including precursor heating will resemble the solutions for lower Mach number shocks. However, the physical details of cosmic-ray heating are not well known, and an in-depth study of cosmic-ray heating is beyond the scope of this paper. Note that in principle additional heating can be incorporated in the equations by parameterizing it in relation to the upstream Mach number and $w$.

Another limitation of the two-fluid approach is that it assumes a steady-state situation and a plane parallel geometry. In a nonsteady-state situation, the shock relations may be influenced by shock acceleration in the past (Drury et al. 1995). Energy flux conservation (Equation (12)) may therefore be violated. Moreover, the highest energy particles may take such a long time between two consecutive shock crossings that the shock velocity has appreciably slowed down. An early discussion of time-dependent effects, as well as the influence of spherical expansion, can be found in Drury et al. (1995; see also Berezhko et al. 1994). Recently, non-steady situations were investigated by Kang et al. (2009), using a kinetic approach, and Schure et al. (2010), using Monte Carlo simulations of test particles coupled to hydrodynamic simulations of spherically expanding SNRs.

In non-steady situations, high compression ratios can be reached, even in the absence of escape of cosmic rays from the system, because in the precursor there is a larger energy flux associated with particles diffusing in the upstream direction than in the downstream direction. In a steady-state situation, this asymmetry is caused by escape of the highest energy particles. In a non-steady-state situation, in which the cosmic-ray particle population builds up (Kang et al. 2009), this asymmetry is caused by the fact that the flow in the upstream direction is from a more energetic particle population that left the sub-shock more recently. It would be interesting to investigate whether one can model non-steady-state shock acceleration in the two-fluid approach by broadening the definition of $\epsilon_{\mathrm{esc}}$ so that it includes this flux asymmetry.

Note that pressure equilibrium (Equation (2)) is probably a good approximation, even in non-steady-state situations. As a result, the relation between $k_{\mathrm{B}} T$ and $w$ is expected to be valid even in non-steady-state situations.

\subsection{Thermodynamics Versus Acceleration Properties}

In the present model, the quantities $\chi_{2}, \chi_{12}, w, \beta$, and $\sigma$ are independent of $\gamma_{\mathrm{cr}}$ and encompass the solutions for particle acceleration based on non-linear kinetic models (Blasi et al. 2005; Vladimirov et al. 2008; Kang et al. 2009; Reville et al. 2009), in which the same equations of the mass flux and the momentum flux conservation are adopted. Typically, the kinetic models depend on two free parameters: the injection efficiency and the maximum momentum, beyond which particles will escape from the system.

As can be seen in Figure 6, our formalism agrees with the results of Blasi et al. (2005), but the solutions by Blasi et al. (2005) all cluster around $w \approx 0.9$ and $\chi_{12}$ around the maximum possible compression factor. The two-fluid approach, however, allows for a broader range of solutions.

In the two-fluid model, $\epsilon_{\mathrm{esc}}$ depends on $\gamma_{\mathrm{cr}}$, which is essentially a free parameter. In the kinetic models, $\epsilon_{\mathrm{esc}}$ is determined self-consistently by the overall hydrodynamic structure of the shock, including the dynamical effects of the accelerated particles. The energy distribution of the accelerated particles then determines $\gamma_{\mathrm{cr}}=1+P_{\mathrm{cr}} / u_{\mathrm{cr}}$, where $P_{\mathrm{cr}}$ is the cosmic-ray pressure and $u_{\text {cr }}$ is the cosmic-ray energy density. Hence, the kinematic models predict that the $\epsilon_{\text {esc }}-w$ diagram should deviate from ours with $\gamma_{\mathrm{cr}}=4 / 3$ and should lie between our curves of $\gamma_{\text {cr }}=4 / 3$ and $5 / 3$. In practice, however, the cosmic-ray adiabatic index, as obtained by the kinetic models, is very close to $\gamma_{\text {cr }}=4 / 3$ for $p_{\max }=10^{5} m c$, as can be seen in Figure 6. For $p_{\max }=10^{2} m c$, we found that a modest increase to $\gamma_{\mathrm{cr}}=1.4$ resulted in a good match between the two-fluid solutions and the kinetic model of Blasi et al. (2005).

Interestingly, the two-fluid solutions presented here indicate that a higher energy flux escape is necessary for $\gamma_{\text {cr }}>4 / 3$, but from the point of view of the spectral energy distribution, the energy escape flux is more difficult to achieve for spectra with spectral energy indices $\Gamma>2\left(N(E) \propto E^{-\Gamma}\right)$, corresponding to $\gamma_{\mathrm{cr}}>4 / 3$. The reason is that for $\Gamma>2$ most of the cosmic-ray energy is contained by mildly relativistic particles, whereas most of the escaping particles will be near the maximum of the energy distribution ( $p_{\max }$ in the Blasi model). Hence, the escaping particles carry away only a small fraction of the internal energy. For $\Gamma<2$ most energy is indeed 

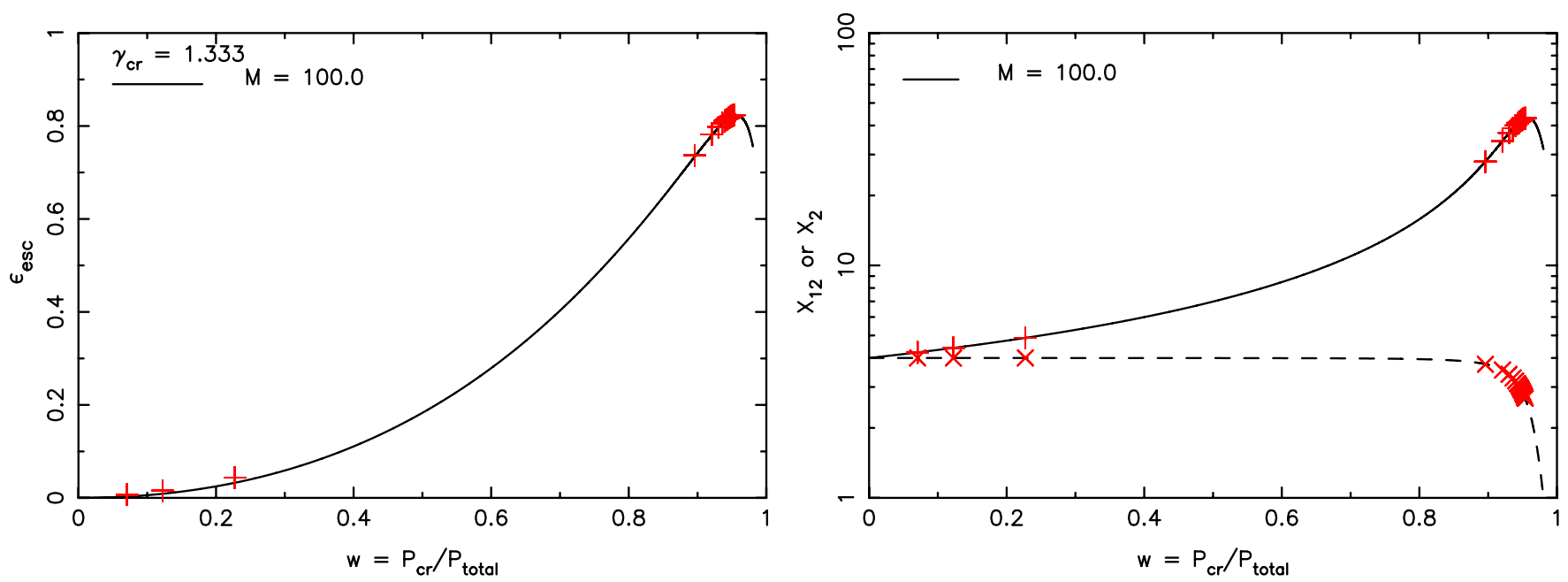

Figure 6. Comparison of the two-fluid model with $M_{0}=100$ with the kinetic model of Blasi et al. (2005) (crosses). The kinetic model was used with $p_{\text {max }}=10^{5} m c$, $\xi=3.5$, and for 20 logarithmically spaced velocities in the range of $10-10^{5} \mathrm{~km} \mathrm{~s}^{-1}$. Left: cosmic-ray escape version post-shock partial cosmic-ray pressure. Right: overall and subshock compression ratio.

(A color version of this figure is available in the online journal.)

concentrated around $p_{\max }$ and the necessary escape flux is easily generated.

So from a thermodynamic point of view, a high fractional cosmic-ray pressure $w$ requires large values of $\epsilon_{\mathrm{esc}}$, which in turn is best achieved with $\gamma_{\text {cr }} \approx 4 / 3$.

\subsection{Application to Observations}

One of the motivations for this work has been the measurements of the downstream temperature of SNRs with reasonably well-known velocities (Helder et al. 2009, 2010). These temperatures were measured using the broad component of the $\mathrm{H} \alpha$ line. This component is due to charge exchange between neutral hydrogen atoms entering the shock and the downstream population of shock-heated protons. Hence, the width of the line is caused by thermal Doppler broadening and reflects the downstream proton temperature. There is some ambiguity as to how to relate the proton temperature to the overall downstream plasma temperature, as the different plasma constituents (electrons, protons, helium, other ions) may not be in thermal equilibrium. However, the proton temperature is always expected to be within a factor of two of the mean plasma temperature.

Using Equation (20) (see also Figure 3) one can easily estimate from a measured temperature and shock velocity what the downstream fractional cosmic-ray pressure is and what the required escape flux is. The only ambiguity that is left for estimating $\epsilon_{\mathrm{esc}}$ is what the effective cosmic-ray adiabatic index is. However, $\gamma_{\mathrm{cr}}$ is not important for determining $w$. For the SNRs under consideration, the shock velocities are well in excess of $1000 \mathrm{~km} \mathrm{~s}^{-1}$. For a typical sound speed in the interstellar medium of $10 \mathrm{~km} \mathrm{~s}^{-1}$ we have $M_{0}>100$. This means that the high Mach number approximation is valid as long as $w \lesssim 0.9$. This appears to be the case for the SNRs considered below.

For the northeastern region of the $\mathrm{TeV}$ gamma-ray-emitting remnant RCW 86, Helder et al. (2009) measured a downstream temperature of $k_{\mathrm{B}} T_{\mathrm{p}}=2.3 \pm 0.3 \mathrm{keV}$ for a measured shock velocity of $V_{s}=6000 \pm 2800 \mathrm{~km} \mathrm{~s}^{-1}$. Nominally, this corresponds to $\beta=0.055$ (see Equation (20)), but given the systematic uncertainties and the ambiguity due to non- equilibration of temperatures $\beta$ could be as high as $\beta=0.31$. Using now Equations (9) and (15) in the limit for high Mach numbers, the measured values of $\beta$ correspond to downstream fractional cosmic-ray pressures and escape fractions of $w=$ $0.81, \epsilon_{\mathrm{esc}}=0.59$ and $w=0.51, \epsilon_{\mathrm{esc}}=0.20$, for $\gamma_{\mathrm{cr}}=4 / 3$ and $\beta=0.055$ and $\beta=0.31$, respectively. For $\gamma_{\mathrm{cr}}=5 / 3$ this is $\epsilon_{\mathrm{esc}}=0.72$ and $\epsilon_{\mathrm{esc}}=0.38$, respectively, with $w$ unchanged.

For the young Large Magellanic Cloud remnant 0509-67.5, Helder et al. (2010) determined the post-shock temperatures in two regions. The most constraining measurement was for the southwestern region for which $k_{\mathrm{B}} T_{\mathrm{p}}=28.7 \mathrm{keV}$ for a conservative velocity estimate of $V_{s}=5000 \mathrm{~km} \mathrm{~s}^{-1}$, corresponding to $\beta=0.58$. This translates into a downstream fractional cosmic-ray pressure of $w=0.29, \epsilon_{\mathrm{esc}}=0.06$ for $\gamma_{\mathrm{cr}}=4 / 3$ or $\epsilon_{\mathrm{esc}}=0.19$ for $\gamma_{\mathrm{cr}}=5 / 3$.

Interestingly, these new estimates of $w$ based on Equations (9) and (15) are not far from the lower limits given by Helder et al. $(2009,2010)$. The reason is that the less constraining relations used by Helder et al. $(2009,2010)$ allow in principle for a higher fractional cosmic-ray pressure by reducing the cosmicray escape flux. The relations derived here do not allow for this possibility. Moreover, the relation that was used to determine the lower limit by Helder et al. $(2009,2010)$ is a limiting case of the shock relations presented here (see Equation (16)).

\subsection{Do Very Efficiently Accelerating Shocks Exist?}

This brings us back to one of the principal issues currently discussed in cosmic-ray physics, namely, do very efficiently accelerating shocks exist? The post-shock temperatures measured by Helder et al. $(2009,2010)$ indicate high values for the cosmic-ray pressure, but not as high as usually found by particle acceleration models, where the efficiency is often found to be close to $90 \%$, with total compression factors as high as $\chi_{12}=70$ (e.g., Blasi et al. 2005). It has been argued by Drury et al. (2009) that such extreme conditions may exist in the prominent TeV gamma-ray source SNR RX J1713.7-3946 (Aharonian et al. 2004). Apart from high gamma-ray luminosity, this idea is based on the lack of detectable thermal X-ray emission. According to Drury et al. (2009), this could be due to a very low 
post-shock plasma temperature, possibly as low as six times the upstream. Alternatively, RX J1713.7-3946 may evolve in a low-density medium, resulting in a low thermal luminosity, as the thermal luminosity scales with the square of the density. The equations derived here allow for very low downstream temperatures. However, the claim by Drury et al. (2009) that the downstream temperature may only be six times the upstream temperature seems unrealistic, as Equations (9) and (15) show that this is only true for unrealistically low Mach numbers. For more realistic Mach numbers for young SNRs, say $M>75$, the downstream temperature is at least 80 times the upstream temperature.

It could be that the young SNRs that we observe are already past their prime as cosmic-ray accelerators, although the cosmicray escape in RCW 86 appears still high enough to explain the cosmic-ray energy production of SNRs. Specifically, it has been argued that in the very early SNR phase/supernova phase cosmic-ray acceleration may be very efficient (e.g., Bell \& Lucek 2001; Vink 2008), see, for example, the case of SN 1993J (Tatischeff 2009). Note that a steady escape of cosmic rays during the evolution of an SNR is favored over a more sudden release of cosmic rays at the end of the SNR evolution, as in the latter case adiabatic losses have lowered the energy of the cosmic rays (Voelk et al. 1984). If one wants to explain the cosmic-ray energy density in the Galaxy by cosmic-ray escaping from young SNR shocks (i.e., $\epsilon_{\mathrm{esc}}=5 \%$, see Section 1), then on average over the lifetime of the SNR a fractional cosmic-ray pressure is necessary of at least $w \approx 30 \%$ for $\gamma_{\mathrm{cr}}=4 / 3$.

\section{SUMMARY AND CONCLUSIONS}

We have shown that for a two-fluid steady-state shock consisting of a thermal gas plus a cosmic-ray component, there exists a unique relation between the downstream fraction of pressure provided by cosmic rays $(w)$ on the one hand, and the energy escape flux normalized to the incoming free-energy flux $\left(\epsilon_{\mathrm{esc}}\right)$ on the other hand. This relation depends on the assumed effective adiabatic index of the cosmic rays and on the overall Mach number. For Mach numbers $M_{0} \lesssim 6$, no cosmicray pressure is allowed. This could be of interest for particle acceleration in clusters of galaxies, since internal cluster shocks tend to be low Mach number shocks, whereas the accretion shocks or cluster formation shock are high Mach number shocks.

This relation can be used to determine $w$ and $\epsilon_{\text {esc }}$ based on measurements of the downstream temperature of the plasma for a known shock velocity. Using this relation, we show that the lower limits on $w$ inferred for shock regions in the supernova remnants RCW 86 and SNR 0509-675 by Helder et al. (2009, 2010) are in fact very close to the actual value for $w$, but we can now determine also $\epsilon_{\mathrm{esc}}$, which is at least $\epsilon_{\mathrm{esc}}=0.2$ for the northeastern region of RCW 86, but only 0.067 for SNR 0509675 (but it may be 0.19 in the unlikely case that the cosmic rays have an effective adiabatic index of $\gamma_{\mathrm{cr}}=5 / 3$ ).
In order to explain the cosmic-ray energy density in the Galaxy by cosmic rays escaping directly from SNR shocks, instead of released at the end of the SNR life, one needs an escape flux of $\epsilon_{\mathrm{esc}} \approx 5 \%$, which requires that on average $30 \%$ of the downstream SNR pressure should be supplied by cosmic rays.

J.V. is supported by a Vidi grant from the Netherlands Science Foundation (NWO). R.Y. is supported by grant-in-aid No. 19047004, No. 21740184, and No. 21540259 from the Ministry of Education, Culture, Sports, Science, and Technology (MEXT) of Japan.

\section{REFERENCES}

Acciari, V. A., et al. 2010, ApJ, 714, 163

Achterberg, A. 2004, in Accretion Discs, Jets and High Energy Phenomena in Astrophysics, 78, ed. V. Beskin et al., 313

Achterberg, A., Blandford, R., \& Periwal, V. 1984, A\&A, 132, 97

Aharonian, F., et al. 2001, A\&A, 370, 112

Aharonian, F. A., et al. 2004, Nature, 432, 75

Aharonian, F., et al. 2008, A\&A, 488, 219

Albert, J., et al. 2007, A\&A, 474, 937

Ballet, J. 2006, Adv. Space Res., 37, 1902

Bamba, A., Yamazaki, R., Yoshida, T., Terasawa, T., \& Katsuji Koyama, K. 2005, ApJ, 621, 793

Bell, A. R. 2004, MNRAS, 353, 550

Bell, A. R., \& Lucek, S. G. 2001, MNRAS, 321, 433

Berezhko, E. G., \& Ellison, D. C. 1999, ApJ, 526, 385

Berezhko, E. G., Yelshin, V. K., \& Ksenofontov, L. T. 1994, Astropart. Phys., 2, 215

Blasi, P., Gabici, S., \& Vannoni, G. 2005, MNRAS, 361, 907

Brown, J. C., Beekman, G., Gray, N., \& MacKinnon, A. L. 1995, A\&A, 299, 629

Caprioli, D., Blasi, P., \& Amato, E. 2009, MNRAS, 396, 2065

Caprioli, D., Blasi, P., Amato, E., \& Vietri, M. 2008, ApJ, 679, L139

Drury, L., Aharonian, F. A., Malyshev, D., \& Gabici, S. 2009, A\&A, 496, 1

Drury, L. O., \& Falle, S. A. E. G. 1986, MNRAS, 223, 353

Drury, L. O., \& Voelk, J. H. 1981, ApJ, 248, 344

Drury, L. O., Voelk, H. J., \& Berezhko, E. G. 1995, A\&A, 299, 222

Ginzburg, V. L., \& Syrovatskij, S. I. 1967, in IAU Symp. 31, Radio Astronomy and the Galactic System, ed. H. van Woerden (Cambridge: Cambridge Univ. Press), 411

Helder, E. A., Kosenko, D., \& Vink, J. 2010, ApJ, 719, L140

Helder, E. A., et al. 2009, Science, 325, 719

Heng, K. 2010, PASA, 27, 23

Hinton, J. A., \& Hofmann, W. 2009, ARA\&A, 47, 523

Kang, H., Ryu, D., \& Jones, T. W. 2009, ApJ, 695, 1273

Reville, B., Kirk, J. G., \& Duffy, P. 2009, ApJ, 694, 951

Schure, K. M., Achterberg, A., Keppens, R., \& Vink, J. 2010, MNRAS, 406, 2633

Tatischeff, V. 2009, A\&A, 499, 191

Vink, J. 2008, in AIP Conf. Ser. 1085, Proc. 4th International Meeting on High Energy Gamma-Ray Astronomy, ed. F. A. Aharonian et al. (Melville, NY: AIP), 169

Vink, J., \& Laming, J. M. 2003, ApJ, 584, 758

Vladimirov, A. E., Bykov, A. M., \& Ellison, D. C. 2008, ApJ, 688, 1084

Voelk, H. J., Drury, L. O., \& McKenzie, J. F. 1984, A\&A, 130, 19

Völk, H. J., Berezhko, E. G., \& Ksenofontov, L. T. 2005, A\&A, 433, 229

Zel'dovich, Y., \& Raizer, Y. P. 1966, Elements of Gasdynamics and the Classical Theory of Shock Waves (New York: Academic) 